\title{
Evaluation of the dosimetric properties of a synthetic single crystal diamond detector in high energy clinical proton beams
}

\author{
A. K. Mandapaka, A. Ghebremedhin, and B. Patyala) \\ Department of Radiation Medicine, Loma Linda University Medical Center, 11234 Anderson Street, \\ Loma Linda, California 92354 \\ Marco Marinelli, G. Prestopino, C. Verona, and G. Verona-Rinati \\ INFN-Dipartimento di Ingegneria Industriale, Università di Roma 'Tor Vergata', Via del Politecnico 1, \\ 00133 Roma, Italy
}

(Received 6 June 2013; revised 19 September 2013; accepted for publication 11 October 2013; published 6 November 2013)

Purpose: To investigate the dosimetric properties of a synthetic single crystal diamond Schottky diode for accurate relative dose measurements in large and small field high-energy clinical proton beams.

Methods: The dosimetric properties of a synthetic single crystal diamond detector were assessed by comparison with a reference Markus parallel plate ionization chamber, an Exradin A16 microionization chamber, and Exradin T1a ion chamber. The diamond detector was operated at zero bias voltage at all times. Comparative dose distribution measurements were performed by means of Fractional depth dose curves and lateral beam profiles in clinical proton beams of energies 155 and $250 \mathrm{MeV}$ for a $14 \mathrm{~cm}$ square cerrobend aperture and $126 \mathrm{MeV}$ for 3, 2, and $1 \mathrm{~cm}$ diameter circular brass collimators. ICRU Report No. 78 recommended beam parameters were used to compare fractional depth dose curves and beam profiles obtained using the diamond detector and the reference ionization chamber. Warm-up/stability of the detector response and linearity with dose were evaluated in a $250 \mathrm{MeV}$ proton beam and dose rate dependence was evaluated in a $126 \mathrm{MeV}$ proton beam. Stem effect and the azimuthal angle dependence of the diode response were also evaluated.

Results: A maximum deviation in diamond detector signal from the average reading of less than $0.5 \%$ was found during the warm-up irradiation procedure. The detector response showed a good linear behavior as a function of dose with observed deviations below $0.5 \%$ over a dose range from 50 to $500 \mathrm{cGy}$. The detector response was dose rate independent, with deviations below $0.5 \%$ in the investigated dose rates ranging from 85 to $300 \mathrm{cGy} / \mathrm{min}$. Stem effect and azimuthal angle dependence of the diode signal were within $0.5 \%$. Fractional depth dose curves and lateral beam profiles obtained with the diamond detector were in good agreement with those measured using reference dosimeters. Conclusions: The observed dosimetric properties of the synthetic single crystal diamond detector indicate that its behavior is proton energy independent and dose rate independent in the investigated energy and dose rate range and it is suitable for accurate relative dosimetric measurements in large as well as in small field high energy clinical proton beams. (C) 2013 American Association of Physicists in Medicine. [http://dx.doi.org/10.1118/1.4828777]

Key words: proton therapy, synthetic diamond detector, relative dosimetry

\section{INTRODUCTION}

The landmark paper proposing the therapeutic use of proton beams was published by Wilson in $1946 .{ }^{1}$ The first hospital-based proton radiotherapy facility was installed at the Loma Linda University Medical Center (LLUMC) in $1990 .^{2}$ Because of the favorable physical characteristics of proton beams over photon and electron beams, the number of centers offering proton therapy is rapidly growing around the world. $^{3,4}$

When dealing with the accuracy of dose-calculation algorithms used in computerized treatment planning systems (TPS), determination and parameterization of the dose distribution of clinical proton beam is very crucial. Proton beams are characterized by strong dose gradients at the treatment volume contour and significant variations in linear energy transfer (LET) and dose rate. Moreover, small field treatments place additional demands on the devices used for their measurement. Since IAEA published the code of practice TRS$398^{5}$ and recommended procedures for ion chamber reference dosimetry for all types of external beams, this has been widely adopted as a standard in proton dosimetry. ${ }^{6}$ ICRU Report No. $78^{7}$ recommends plane parallel ionization chambers (ICs) ${ }^{8}$ as reference instrument for depth dose measurements in clinical proton beams, but small volume thimble ionization chambers 9 and diamond detectors ${ }^{10-12}$ can also be used. In particular, IAEA TRS-398 code of practice recommends the use of detectors with a better spatial resolution (e.g., mini-chambers, diodes, diamonds) if the proton field size is smaller than twice the diameter of the cavity of the plane parallel chamber. The suitability of such detectors has to be preverified by test comparison with plane parallel IC at larger field size. ${ }^{5}$ 
Microchambers have widespread use in small field dosimetry, but they do have limitations with regards to their sensitivity. ${ }^{13,14}$ Film dosimetry provides a good spatial resolution, but its disadvantages are LET and energy dependency. In addition, dose cannot be obtained from optical density in mixed fields and offline analysis is required. ${ }^{6,15}$ GafChromic EBT film has been used for relative dosimetry in proton beams ${ }^{16}$ but it requires LET dependent correction factors. A BANG3- Pro2 polymer gel was evaluated for dosimetry in proton beams. ${ }^{17}$ Quantitative dose distribution comparisons to treatment planning system calculations show that $>97 \%$ of the gel dose maps pass the $3 \% / 3 \mathrm{~mm}$ gamma criterion. However, Gel dosimetry requires a CT scanner for the dose readout process and also requires a dark environment in the scanning area to minimize effects of stray light which can contribute to the photodetector signal. An inorganic scintillating detector was also studied for proton beam dosimetry verification. ${ }^{18}$ Small volume solid state detectors, such as silicon diodes and diamond detectors, seem to be the most promising instruments for relative dosimetric measurements of clinical proton beams. ${ }^{6,12,19}$

Due to its outstanding properties, such as high radiation hardness, near tissue equivalence, small size, and low leakage current, diamond has long been considered an ideal material for the construction of small volume high-resolution radiation detectors. The suitability of natural diamond-based radiation therapy detectors from PTW-Freiburg for dosimetric measurements in clinical proton beams has been discussed in various publications. ${ }^{10-12,20,21}$ The diamond detectors used in those studies showed a significant dose rate dependence and a substantial dependence on beam quality. The need of specific correction factors for each individual diamond detector used, accounting for dose rate dependence of detector response and LET dependence of calibration factor, was pointed out. ${ }^{20}$

Recently, the feasibility of high performance radiation therapy synthetic single crystal diamond detectors (SCDDs) grown by means of CVD technique at the University of Rome
"Tor Vergata" laboratories was successfully demonstrated for photon as well as electron beams. ${ }^{22,23}$ In this work the dosimetric performance of one such detector was evaluated in high-energy clinical proton beams at Loma Linda University Medical Center. Relative dosimetric properties of the SCDD in measuring Fractional depth dose (FDD) and beam profiles were compared to those of reference ICs by means of analysis of parameters as recommended in ICRU Report No. 78. Dose linearity, dose rate dependence, reproducibility of the diamond detector signal, detector stem effect, and the diode azimuthal angular response were also evaluated.

\section{MATERIALS AND METHODS}

\section{A. Horizontal beam line at Loma Linda University Medical Center}

Commercial treatment systems for proton therapy are available using both synchrotron and cyclotron accelerators. The synchrotron system at LLUMC was designed by Fermi lab and upgraded and marketed by Optivus Proton Therapy, Inc. The parameters of the synchrotron used at LLUMC are well documented. ${ }^{24}$ All the experiments reported in this study were done in the fixed horizontal beam line (HBL) capable of treating large as well as small fields. The virtual source to axis distance of the machine is $250 \mathrm{~cm}$.

Figure 1 shows different components in the HBL nozzle. Several detectors are placed in the beam path in order to monitor the beam. Going downstream in the beam direction, the following detectors can be found: secondary emission monitor (SEM), multiwire ion chamber (MWIC), three transmission ion chambers (Tic1, Tic2, and Tic3). The SEM triggers the beam off if an unusually high beam current is detected. The MWIC monitors the initial beam profile for beam diagnostics purpose. Tic1 -3 are segmented parallel plate ion chambers and are used to check the focus of the beam. Tic3 deserves further explanation. It consists of two independent planes of ion chambers. Plane 1 has 3 concentric ring

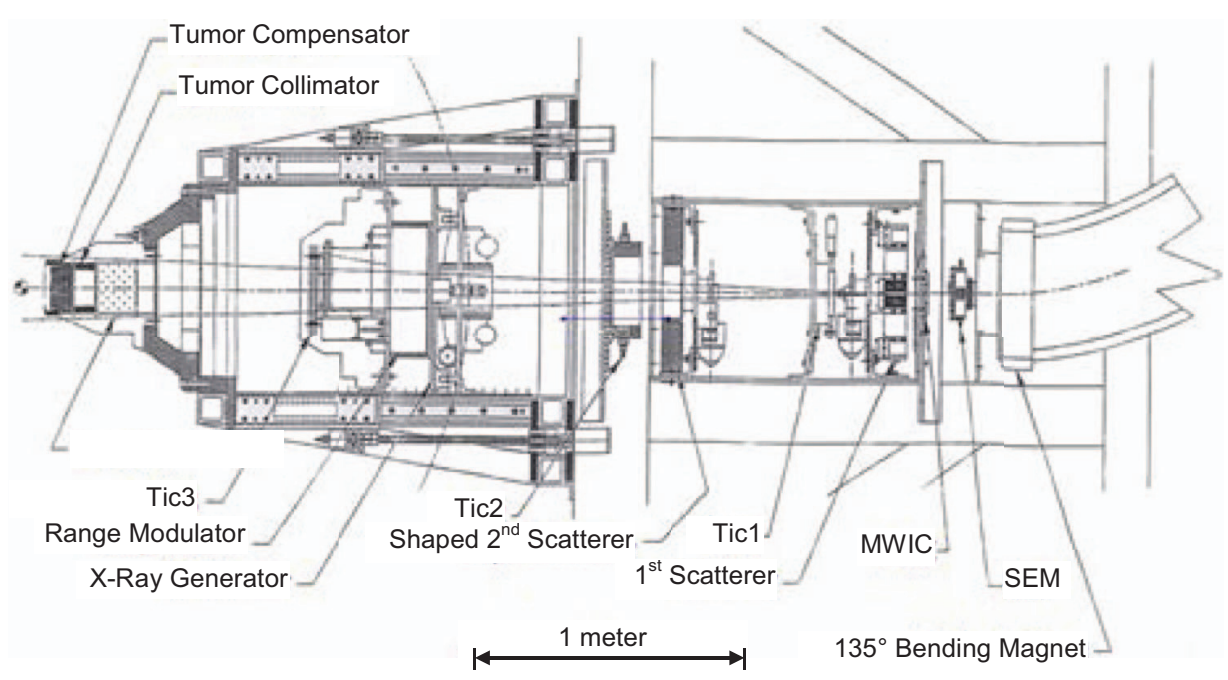

FIG. 1. Schematic of the horizontal beam line (HBL) nozzle which shows different detectors in the beam line. 
detectors (central spot, ring 1, ring 2) and plane 2 consists of 317 detectors arranged in a square pattern. Tic3-ring1 is the primary internal dosimeter in HBL. During the experiments, the proton beam was centered at all times and the transmission ion chambers monitored the beam centering. The machine output is calibrated on a daily basis. The standard output calibration setup for a particular beam energy using large fields consists of a $14 \mathrm{~cm}$ square cerrobend aperture for beam collimation and a $60 \mathrm{~mm}$ range modulator wheel. The counts from Tic3-ring1 correspond to the charge collected by the ion chamber which is placed at the center of modulation of the beam, which in-turn corresponds to the dose delivered by the machine in cGy for the standard setup. A modulator wheel, inserted in the beam path, is used to spread out the pristine Bragg peak. An unmodulated beam was used in the current experiments, except where explicitly noted otherwise. The available clinical beam energies in HBL for patient treatments are 126, 155, 200, and $250 \mathrm{MeV} .126 \mathrm{MeV}$ protons are exclusively used for stereotactic radiosurgery treatments.

The dosimetric properties of the diamond detector were investigated in 126, 155, and $250 \mathrm{MeV}$ proton beams. Beam collimation was achieved using cerrobend apertures for large fields and brass collimators for small stereotactic fields. A 14 $\mathrm{cm}$ square aperture (quality assurance or QA aperture) was used with 155 and $250 \mathrm{MeV}$, whereas 3, 2, and $1 \mathrm{~cm}$ diameter circular brass collimators were used with $126 \mathrm{MeV}$ protons.

\section{B. Diamond detector}

The SCDD tested in this study was based on a similar design of a diamond detector investigated in a previous work. ${ }^{22}$ The basic device structure consists of a multilayered synthetic single crystal p-type diamond/intrinsic diamond structure fabricated at Roma "Tor Vergata" University laboratories by means of a two-step microwave plasma enhanced chemical vapor deposition process on a commercial low-cost $3.0 \times 3.0 \times 0.3 \mathrm{~mm}^{3}$ high-pressure high-temperature single crystal diamond substrate. Such deposition was followed by thermal evaporation of a circular $2.2 \mathrm{~mm}$ in diameter rectifying metallic contact on the intrinsic diamond surface and an ohmic contact on the p-type diamond. Due to the builtin potential at the metal/intrinsic diamond interface the device works as a Schottky barrier photodiode and is operated with no external bias voltage applied. The detector sensitive volume was about $0.0038 \mathrm{~mm}^{3}$, determined by the depletion region extending through the whole thickness of the 1.0 $\pm 0.1 \mu \mathrm{m}$ thick intrinsic diamond layer below the circular Schottky contact. A thorough study of the device fabrication process, of its physical properties, and detection mechanism was reported in a previous study. ${ }^{25}$

The SCDD was finally embedded in a polymethylmethacrylate (PMMA) waterproof cylindrical housing, $8 \mathrm{~mm}$ in diameter and $27 \mathrm{~mm}$ long, filled by a two-component epoxy resin and connected to a low noise triaxial cable/connector. The diamond surface was located $1.5 \mathrm{~mm}$ below the top surface of the housing. At the end of the encapsulation process,

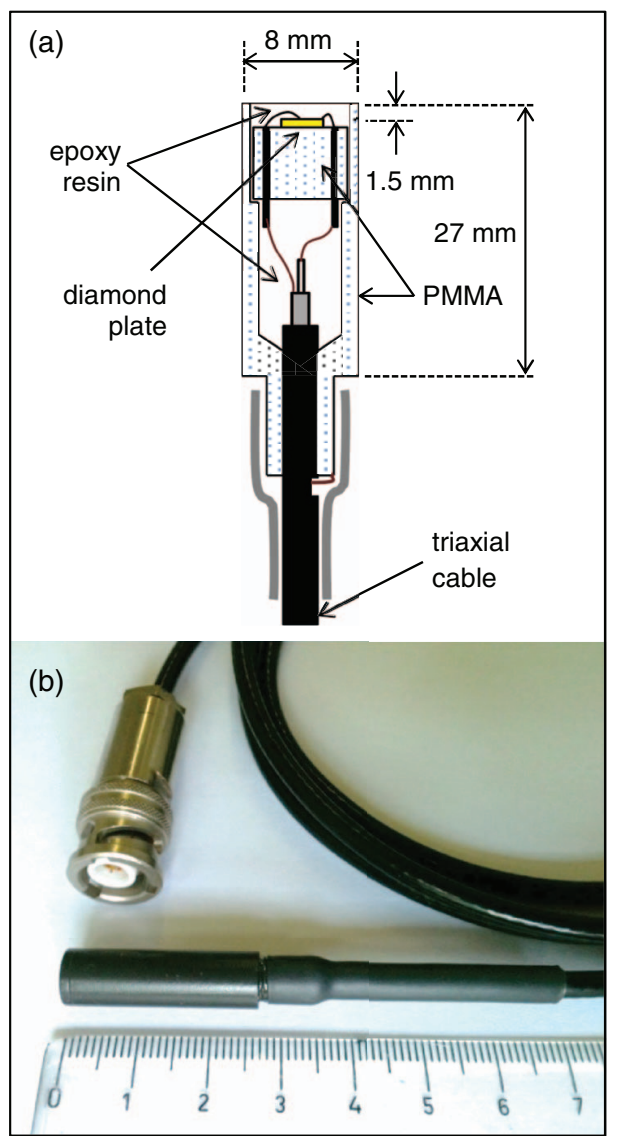

FIG. 2. (a) Sketch of the synthetic single crystal diamond detector and electrical connections inside PMMA housing filled with epoxy resin, and (b) photo of the triaxial cable and connector and of the graphite coated waterproof capsule.

the cylindrical probe was covered with a conductive graphite based lacquer used as a shield to reduce the noise. A sketch of the SCDD and of its electrical connections to the triaxial cable inside the epoxy resin filled PMMA waterproof housing is shown in Fig. 2(a). Figure 2(b) shows a picture of the graphite coated capsule and of the triaxial cable/connector. For the measurements reported in this study the detector long axis was always parallel to the beam direction, unless noted otherwise. The SCDD measurement point was assumed to be at the center of the top surface of the intrinsic diamond layer, $1.5 \mathrm{~mm}$ below the detector tip.

\section{C. Reference detectors, electrometers, and water tank}

The dosimetric properties of the SCDD were assessed by comparison with three commercially available reference ionization chambers: a Markus parallel plate ionization chamber (type 23343, PTW-Freiburg), an Exradin A16 microion chamber (REF 92726, Standard Imaging), and Exradin T1a ion chamber (S.No. XG101021).

The type 23343 Markus chamber (MK-IC in the following) is designed specifically for absolute dosimetry in high-energy electron beams. The chamber has a nominal sensitive volume 
of $55 \mathrm{~mm}^{3}$ with a radius of $2.65 \mathrm{~mm}$ and a depth of $2 \mathrm{~mm}$. The entrance window is a thin $30 \mu \mathrm{m}$ polyethylene foil with a conductive graphite coating. When used in a water phantom, a $0.87 \mathrm{~mm}$ thick PMMA protective cap is screwed onto the chamber and the physical effective point of measurement is $1.06 \mathrm{~mm}$ below the surface of the protective cap. Following manufacturer recommendations, the chamber plane was placed perpendicular to the proton beam direction and a polarizing voltage of $+300 \mathrm{~V}$ was applied. The MK-IC was used as a reference detector for PDD measurements in large field proton beams.

The Exradin A16 ion chamber (A16-IC in the following) is a microionization chamber designed primarily for small field dosimetry in intensity modulated radiation therapy and stereotactic radiosurgery applications. The chamber has a collecting volume of $7 \mathrm{~mm}^{3}$, with a collector diameter of $0.3 \mathrm{~mm}$ and a collector length of $1.27 \mathrm{~mm}$. The centroid of collecting volume is approximately $1.7 \mathrm{~mm}$ from the tip of chamber. The A16-IC was used as a reference detector for PDD measurements in small fields and to obtain lateral beam profiles for all field sizes. A16-IC was oriented with its axis perpendicular to the beam direction and a polarizing voltage of $+300 \mathrm{~V}$ was applied.

The Exradin T1a ion chamber (T1a-IC in the following) is a thimble ionization chamber. It has a collection volume of $0.053 \mathrm{~cm}^{3}$, with a collector diameter of $1.0 \mathrm{~mm}$. The T1aIC was used as a reference detector for dose-rate dependent measurements. The T1a-IC was operated at $+300 \mathrm{~V}$.

A Cardinal Health electrometer (model 35040) and a Keithley electrometer (Model 6517A) were used for all charge measurements. The electrometers were calibrated at an ADCL laboratory.

Two water tanks, made in-house, were used in this study. The first tank has dimensions of $50 \times 25 \times 25 \mathrm{~cm}^{3}$ (tank1). On one side, it has a $5.92 \mathrm{~mm}$ thick and $170 \mathrm{~mm}$ diameter circular entrance window made of polycarbonate. The water equivalent thickness (WET) of the polycarbonate entrance window is $6.81 \mathrm{~mm}$. The other walls of tank1 are $8.6 \mathrm{~mm}$ in physical thickness $($ WET $=9.9 \mathrm{~mm}$ ) and made of acrylic. The second tank has dimensions of $23 \times 23 \times 23 \mathrm{~cm}^{3}$ (tank2). On one side, it has a $1 \mathrm{~mm}$ thick and $50 \mathrm{~mm}$ diameter circular acrylic entrance window. The other walls of the tank are $9 \mathrm{~mm}$ thick acrylic. Tank1 was used for PDD measurements in large fields, dose rate dependence, and linearity with dose. Tank2 was used for all beam profile measurements and PDD measurements in small fields. Software developed in-house was used to control the detector position in the water tanks. The software is capable of moving the detector in increments of $0.1 \mathrm{~mm}$. The water equivalent thickness of the tank entrance window was always considered when defining the absolute position of the detector active area in the water tank.

\section{D. Measurements details}

All measurements were performed in the HBL treatment room at James M. Slater, M.D. Proton Treatment and Research Center at LLMUC. The list of parameters for the measurements performed is summarized in Table I.

The detectors were carefully lined up to be in the centre of the proton beam, by taking beam-line and lateral $\mathrm{x}$-rays images with a $1 \mathrm{~cm}$ circular brass collimator in place. The position of the detectors was adjusted until the centre of the detector coincides with the centre of the $1 \mathrm{~cm}$ aperture on the $\mathrm{x}$-ray image. The detector position in the beam was verified at the proximal and the distal edges of the PDD, to make sure the detector was travelling in a straight line along the beam axis for these measurements.

The active area of the detector was placed at a known water equivalent depth and this depth was set in the software that controlled the detector movement. Before taking any measurements the detectors were allowed to reach thermal equilibrium within the water-tank.

The warm-up/stability of the SCDD was tested in a $250 \mathrm{MeV}$ proton beam with the detector placed at a depth of $200 \mathrm{~mm}$ in water. For a constant backup counter setting approximately corresponding to a dose of $15 \mathrm{cGy}$, the charge $\left(\mathrm{M}_{\mathrm{SCDD}}\right)$ measured by the SCDD and the counts from Tic3ring1 were recorded. This procedure was repeated 6 times and the ratio of $\mathrm{M}_{\mathrm{SCDD}}$ to the Tic3-ring1 counts was calculated for each step.

Dose linearity of the SCDD was measured in a $250 \mathrm{MeV}$ proton beam. The active area of the detector was placed at a water equivalent depth (WED) of $120 \mathrm{~mm}$ and $60 \mathrm{~mm}$ modulation wheel was placed in the beam path to get a $60 \mathrm{~mm}$

TABLE I. Summary of the main experimental parameters for the measurements reported in this study.

\begin{tabular}{|c|c|c|c|c|c|c|}
\hline Type of measurement & $\begin{array}{c}\text { Beam energy } \\
(\mathrm{MeV})\end{array}$ & $\begin{array}{c}\text { Modulation } \\
\text { wheel }\end{array}$ & $\begin{array}{c}\text { Nozzle } \\
\text { setting }(\mathrm{mm})\end{array}$ & $\begin{array}{l}\text { Size of aperture } \\
(\mathrm{cm})^{\mathrm{a}}\end{array}$ & $\begin{array}{l}\text { Position of tank } \\
\text { entrance }(\mathrm{cm})^{\mathrm{b}}\end{array}$ & $\begin{array}{l}\text { Reference } \\
\text { detector }\end{array}$ \\
\hline PDD & 155 & 0 & 480 & 14 & $10.5, \mathrm{u}$ & MK-IC \\
\hline PDD & 250 & 0 & 320 & 14 & $10.5, \mathrm{u}$ & MK-IC \\
\hline PDD & 126 & 0 & 220 & $1,2,3$ & $2, \mathrm{~d}$ & A16-IC \\
\hline Profiles & 155 & 0 & 480 & 14 & $5, \mathrm{u}$ & A16-IC \\
\hline Profiles & 250 & 0 & 320 & 14 & $5, \mathrm{u}$ & A16-IC \\
\hline Profiles & 126 & 0 & 220 & $1,2,3$ & $2, \mathrm{~d}$ & A16-IC \\
\hline Dose linearity & 250 & 60 & 320 & 14 & $10.5, \mathrm{u}$ & None \\
\hline Dose rate dependence & 126 & 30 & 220 & 3 & $2, \mathrm{~d}$ & T1a-IC \\
\hline
\end{tabular}

${ }^{\mathrm{a}} 14 \mathrm{~cm}$ square cerrobend aperture; $1,2,3 \mathrm{~cm}$ in diameter circular brass collimators.

${ }^{\mathrm{b}} \mathrm{u}=$ upstream from isocenter; $\mathrm{d}=$ downstream from the end of the stereotactic cone. 
spread out Bragg peak (SOBP). For each successive beam run the backup counter setting was increased linearly, while the Tic3-ring 1 counts and the charge measured by the SCDD were recorded. The dose delivered at the position of the SCDD was calculated from the Tic3-ring1 counts. The Tic3ring 1 counts were converted to dose in cGy at the centre of modulation (COM) using the machine daily output calibration report. The absolute dose at the depth of measurement was calculated as the product of the dose at the COM and the percentage depth dose at that depth. The linearity of SCDD response with dose was investigated in the 0.515-5.14 Gy dose range. During the dose dependence measurements the beam was run in the maximum ring current capacity (i.e., maximum dose rate).

Dose-rate dependence of SCDD was studied in a modulated $126 \mathrm{MeV}$ proton beam using a $30 \mathrm{~mm}$ SOBP and a $3 \mathrm{~cm}$ circular brass collimator. This energy was selected for dose rate measurements because higher dose rates and larger dose rate variations are achievable at low energy with a small aperture. The dose-rate was varied from 85 to $300 \mathrm{cGy} / \mathrm{min}$. The protons from the accelerator are produced in the form of "spills." Each spill of protons will last for $2.2 \mathrm{~s}$. The accelerator operator can maximize the number of protons up to 2.2 $\times 10^{10}$ protons/spill. This manipulation of the number of protons/spill is a direct correlation to the number of particles extracted, which is how the dose-rate is varied. The active area of the SCDD was placed in the centre of the beam at a WED of $85 \mathrm{~mm}$, which corresponds to the COM depth of this particular beam arrangement. An Exradin T1 ionization chamber was taped outside the water tank entrance window, near the edge of the beam but making sure that its active area was completely in the beam path. Since the T1 ionization chamber is already known to be dose-rate independent, it was used as a reference for measurements with SCDD. For each measurement, charges measured by SCDD and by the T1 ionization chamber were noted and so were the Tic3-ring1 counts and the number of beam spills. For good statistics, five readings of the charge measured by SCDD and T1 were taken at each dose-rate setting and the average of the ratio of the SCDD reading to the $\mathrm{T} 1$ reading was calculated.

Stem effect measurements were performed in a modulated $155 \mathrm{MeV}$ proton beam using a $60 \mathrm{~mm} \mathrm{SOBP}$ and a $14 \mathrm{~cm}$ square aperture with a custom $10 \times 2 \mathrm{~cm}$ opening. The diamond detector was placed in a water tank at a depth of the centre of the SOBP, which is approximately $10 \mathrm{~cm}$. With the detector axis moving parallel to the long axis of the treatment field (orientation 1), charge and the Tic3-ring1 counts were measured at several points in the field. Then the detector was rotated such that now its axis is perpendicular to the long axis of the treatment field (orientation 2). With the detector positioned at the same points as before, charge measured and Tic3 counts were noted. The reproducibility of the detector position for the two orientations was verified using the beam-line $\mathrm{X}$-ray images. The ratio of SCDD charge to Tic3 counts was then calculated both for orientation $1\left(\mathrm{R}_{1}\right)$ and orientation 2 $\left(R_{2}\right)$ measured. Essentially, different lengths of the diode stem were irradiated in the first orientation, while a same detector length was irradiated in the second orientation. The stem ef- fect was finally evaluated by studying the ratio $R_{1} / R_{2}$ as function of the device position in the beam.

Azimuthal angle dependence of the diode signal was evaluated in the same proton beam conditions as for the stem effect, but now with the diode axis perpendicular to the beam direction. The SCDD charge and the Tic3-ring1 counts were noted for the detector positioned at $0^{\circ}, 90^{\circ}, 180^{\circ}$, and $270^{\circ}$ with detector being rotated along its axis. Due to the detector holder limitations, the authors were unable to perform polar angle dependent study. Prior study ${ }^{22}$ was done regarding polar angle dependence of the diode signal for a similar diode detector in photon beams and we believe that the results would be similar in proton beams.

Fractional depth dose and cross-plane beam profiles were acquired for 126, 155, and $250 \mathrm{MeV}$ beams. All beam profiles were acquired with the detector positioned at a WED of $5 \mathrm{~cm}$. As reported in Table I, the three circular brass beam collimators were used in the $126 \mathrm{MeV}$ beam, whereas the $14 \mathrm{~cm}$ square cerrobend aperture was used with the 155 and $250 \mathrm{MeV}$ proton beams. Fractional depth doses were measured in $5 \mathrm{~mm}$ increments in the proximal flat region, $1 \mathrm{~mm}$ increments in the high dose gradient area distal and proximal to the Bragg peak, and in $0.5 \mathrm{~mm}$ increments near the Bragg peak. All the FDD curves were normalized to the plateau at the entrance distance of $10 \mathrm{~mm}$.

Beam profiles for 155 and $250 \mathrm{MeV}$ proton beams were acquired in $5 \mathrm{~mm}$ increments outside the penumbra region, $1 \mathrm{~mm}$ increments in the penumbra region and the high dose gradient region, and $3 \mathrm{~mm}$ increments in the central nearly flat region. Profiles for the smaller circular fields were acquired in $1 \mathrm{~mm}$ increments. All measured profiles were normalized at the maximum dose value. Parameters for analysis of depth dose curves and lateral beam profiles were obtained from ICRU Report No. 78, ${ }^{7}$ where parameterizations of Gall et al. ${ }^{26}$ and Gottschalk ${ }^{27}$ are recommended to characterize lateral dose and depth dose distributions, respectively.

\section{RESULTS AND DISCUSSION}

\section{A. Warm-up/stability, dose linearity, dose-rate dependence, stem effect, and azimuthal angle dependence}

A warm-up irradiation procedure in the $250 \mathrm{MeV}$ proton beam was performed before each measurement session to verify the SCDD signal stability and the eventual need of a warmup procedure. In Fig. 3 results of one of such warm-up procedure are plotted. Deviations of less than $0.5 \%$ are observed over the warm-up period.

The linearity with dose in the $250 \mathrm{MeV}$ proton beam of the SCDD is shown in Fig. 4. Figure 4(a) shows the charge measured by SCDD as a function of the delivered dose. A very good linear behavior was found, with the $\mathrm{R}^{2}$ parameter of the linear best fit equal to 1 and a precision of $10^{-6}$. A sensitivity of $0.677 \mathrm{nC} / \mathrm{Gy}$ was calculated from the slope of the linear fit. Deviations from linearity of the SCDD response are shown in Fig. 4(b) as percentage deviation of the measured charge to delivered dose ratio with respect to the value at the 


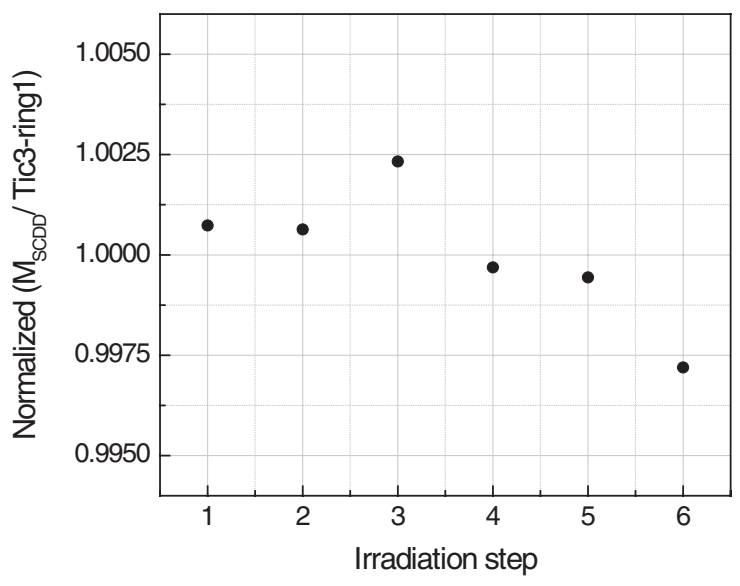

FIG. 3. Warm-up of the SCDD in a $250 \mathrm{MeV}$ proton beam. Normalized ratios of the SCDD measured charge and Tic3-ring1 counts for consecutive irradiation steps are reported. Each point was normalized to the average value.

maximum dose. Deviations of less than $\pm 0.5 \%$ are observed, which are well within experimental error.

Figure 5 shows the dose rate dependence of the SCDD in a $126 \mathrm{MeV}$ proton beam. Percent deviation of the SCDD to $\mathrm{T} 1$ charge ratio $\mathrm{R}$ at each dose rate setting with respect to the average value $\langle R\rangle$ is plotted as a function of dose rate. The error bar in the plot is the ratio of the standard deviation of the SCDD to T1 charge ratio to the average of the SCDD to T1 charge ratio at the lowest dose rate setting, which happened to be the largest standard deviation of all dose rate settings. The dose rate of the beam was calculated using the measured Tic3ring1 counts and the number of spills delivered in the measurement interval. A maximum deviation of $0.5 \%$ was found

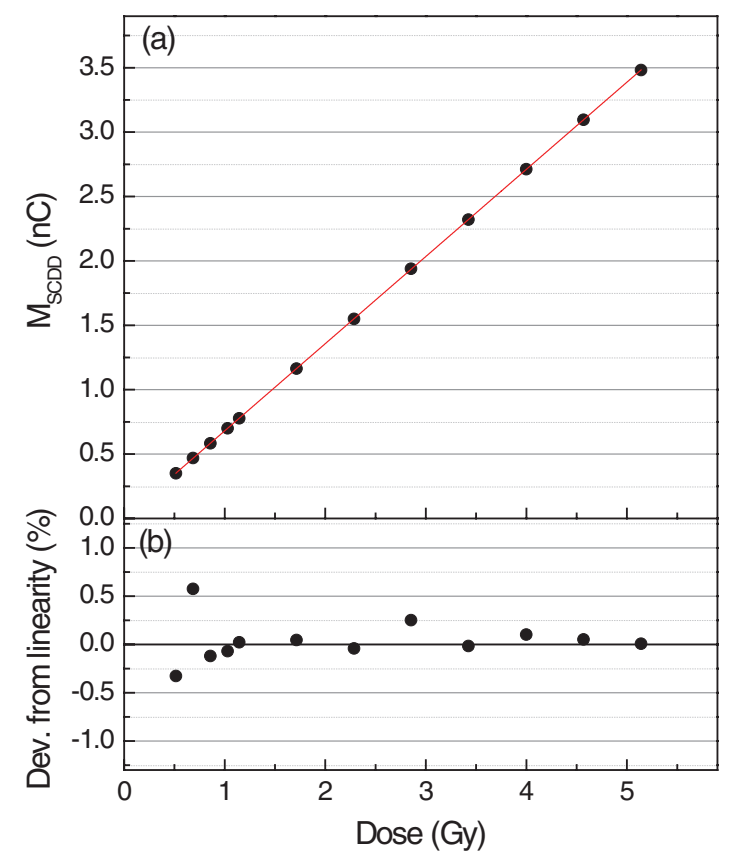

FIG. 4. (a) Measured SCDD charge $\mathrm{M}_{\mathrm{SCDD}}$ vs delivered dose under irradiation with the $250 \mathrm{MeV}$ proton beam. (b) Percentage deviation of the $\mathrm{M}_{\mathrm{SCDD}}$ to delivered dose ratio with respect to the value at the maximum dose.

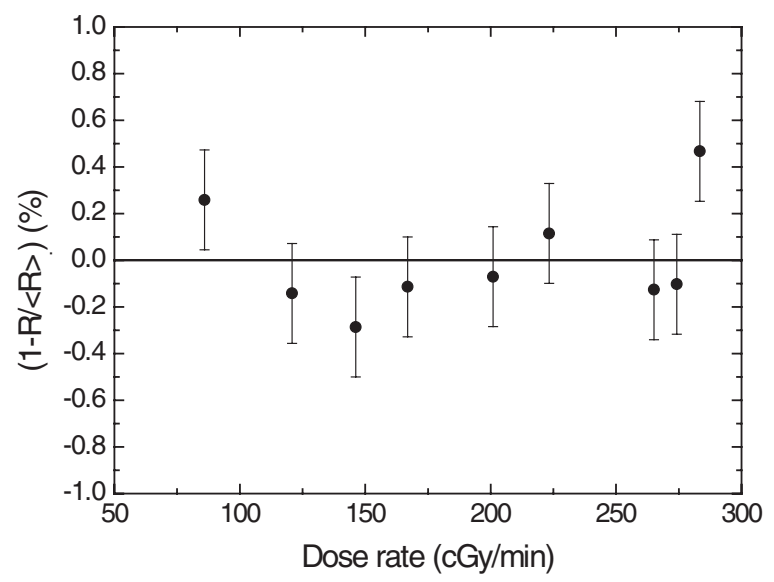

FIG. 5. Dose rate dependence of the SCDD response. Percentage deviation of the SCDD to T1a-IC measured charge ratio is reported as a function of the dose rate. $\mathrm{R}$ is the ratio of the measured SCDD to T1a-IC charge at a particular dose rate. $\langle R\rangle$ is the average of $R$ values.

in the investigated dose rate range, indicating negligible dose rate dependence.

Stem effect data are shown in Fig. 6, where the ratio $R_{1} / R_{2}$ is reported as a function of the length of stem irradiated. A maximum deviation of $0.5 \%$ was found.

\section{B. Fractional depth dose curves}

Central axis FDD curves measured by SCDD and reference ionization chambers are shown in Figs. 7 and 8. All curves are normalized at $10 \mathrm{~mm}$ depth in water in the plateau region. In some cases, a best fit in the entrance region by an exponential growth function was used to extrapolate depth dose values. No corrections were applied for dose-rate effects or for water-to-detector material stopping power ratios. Figure 7 shows FDD curves from SCDD and MK-IC in 155

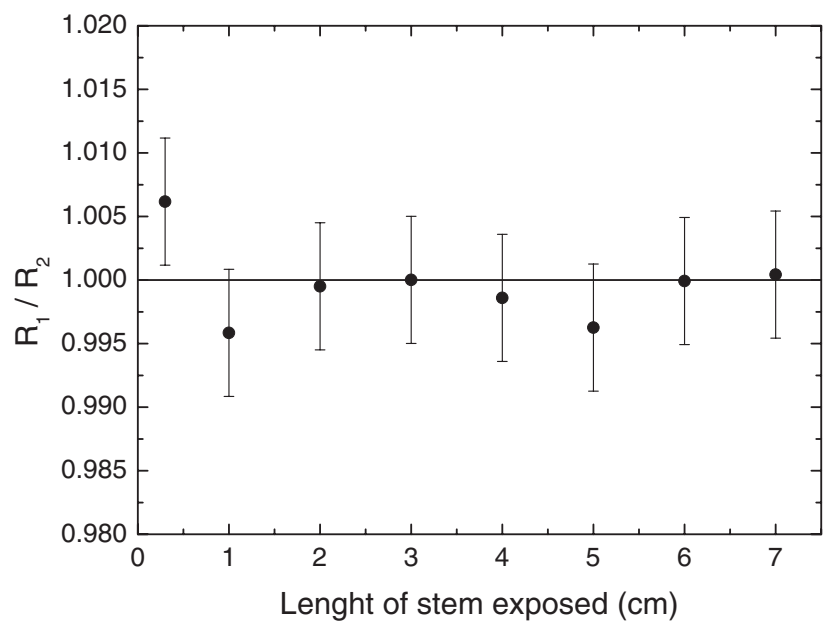

FIG. 6. Stem effect. Plot of stem correction factor (R1/R2) as a function of stem length. R1 is the reading with the diode detector axis moving parallel to the long axis of the treatment field. $\mathrm{R} 2$ is the reading with the detector rotated such that now its axis is perpendicular to the long axis of the treatment field. 


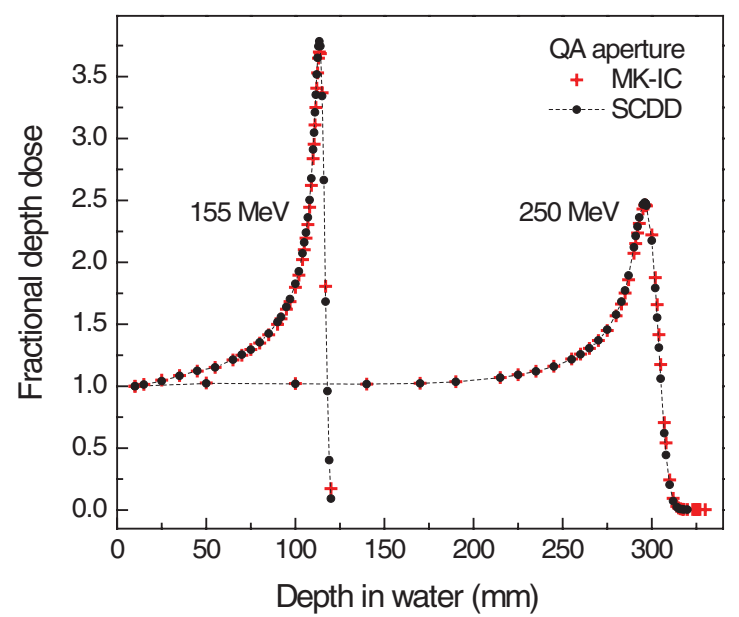

FIG. 7. Fractional depth doses measured by SCDD and MK-IC in 155 and $250 \mathrm{MeV}$ proton beams using $14 \mathrm{~cm}$ square QA aperture. PDD curves are normalized at plateau $(10 \mathrm{~mm})$.

and $250 \mathrm{MeV}$ proton beams, with the $14 \mathrm{~cm}$ QA aperture. Depth dose curves for the 1,2 , and $3 \mathrm{~cm}$ diameter circular brass collimators measured in the $126 \mathrm{MeV}$ proton beam by SCDD and A16-IC are reported in Fig. 8. The water equivalent depth of the A16-IC was defined by comparing the PDD obtained with Markus chamber and the A16-IC in a $250 \mathrm{MeV}$ beam using the QA aperture. An excellent agreement between the FDD curves using SCDD and the two reference ICs for all investigated beam apertures and proton energies is clearly observed. A detailed analysis of depth dose curves was performed by using parameters recommended by ICRU Report No. $78^{7}$ : (i) depth of penetration d' ${ }_{90}$, defined as the depth in water on the central beam axis in which distal-90\% of maximum dose is obtained, and (ii) the distal-dose fall off (DDF), defined as the distance on the central beam axis between points representing distal-80\% and distal-20\% of maximum dose in the decreasing part of the Bragg peak. The computed quantities are reported in Table II. Small negative differences between $\mathrm{d}_{90}^{\prime}$ values from SCDD and ICs PDD curves can be observed for all the proton energies and apertures investigated. Such differences may be attributed either to a positioning error or to an uncertainty in the detector effective point of measurement. A good agreement is also observed

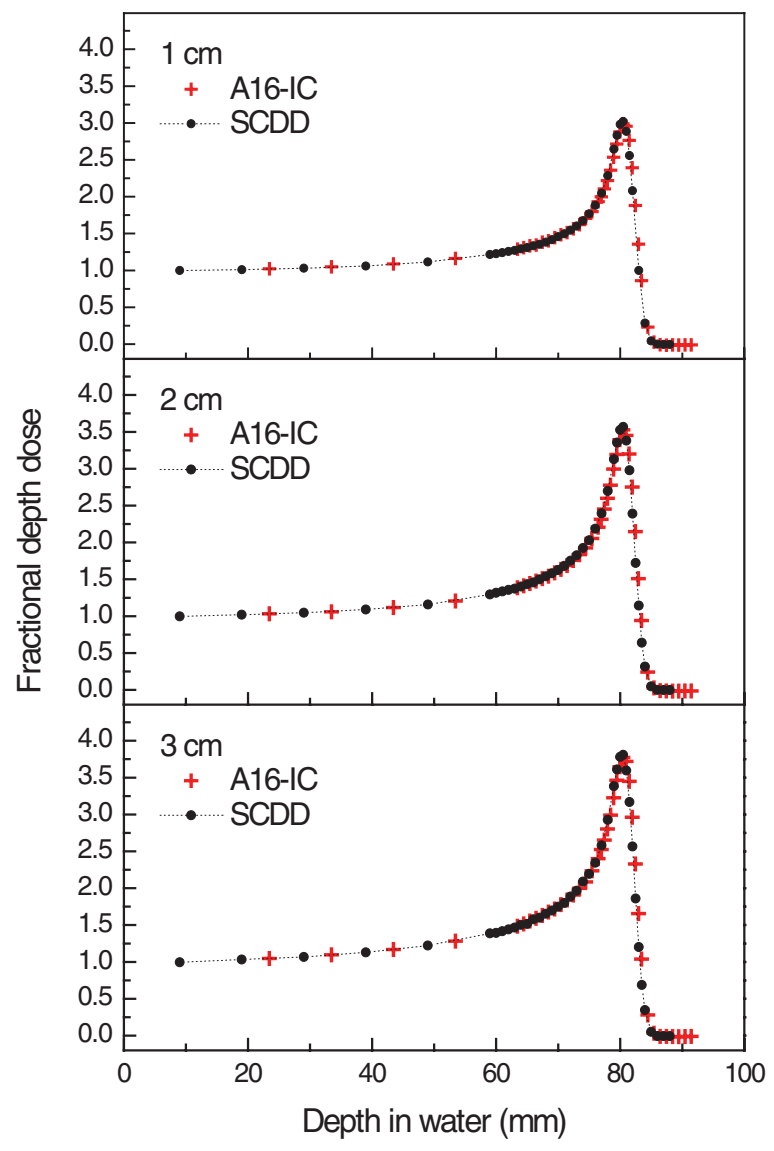

FIG. 8. Fractional depth doses measured by SCDD and A16-IC in the $126 \mathrm{MeV}$ proton beam with $1,2,3 \mathrm{~cm}$ diameter circular brass collimators. The curves are normalized at plateau $(10 \mathrm{~mm})$.

between the DDF values derived for SCDD and the reference ICs.

To verify the energy and LET dependence of the SCCD, the peak-to-plateau ratios for the various PDD curves were also evaluated and reported in Table II. No significant deviations in depth dose curves measured by small volume SCDD with respect to those from reference ICs were observed, indicating the absence of LET dependence of the SCDD in the investigated energy range. In particular, variation in peak-toplateau ratio below $2.3 \%$ can be observed among PDD curves from SCDD and ICs. In all cases the Bragg peak measured

TABLE II. PDD analysis parameters for the depth dose curves shown in Figs. 6 and 7.

\begin{tabular}{|c|c|c|c|c|c|c|c|c|c|}
\hline \multirow{2}{*}{$\begin{array}{l}\text { Proton energy - } \\
\text { size of aperture }\end{array}$} & \multicolumn{3}{|c|}{$\mathrm{d}_{90}^{\prime}(\mathrm{mm})$} & \multicolumn{3}{|c|}{ Distal-dose falloff (mm) } & \multicolumn{3}{|c|}{ Peak-to-plateau ratio } \\
\hline & SCDD & IC & difference & SCDD & $\mathrm{IC}$ & Difference & SCDD & $\mathrm{IC}$ & Rel. diff. $(\%)^{\mathrm{b}}$ \\
\hline $155 \mathrm{MeV}-14 \mathrm{~cm}$ & 114.8 & 115.0 & -0.2 & 2.9 & 3.4 & -0.5 & 3.784 & 3.698 & 2.3 \\
\hline $250 \mathrm{MeV}-14 \mathrm{~cm}$ & 299.4 & 300.0 & -0.6 & 6.7 & 6.9 & -0.2 & 2.477 & 2.463 & 0.0 \\
\hline $126 \mathrm{MeV}-1 \mathrm{~cm}$ & 81.2 & 81.5 & -0.3 & 1.9 & 1.9 & -0.0 & 3.020 & 2.990 & 1.0 \\
\hline $126 \mathrm{MeV}-2 \mathrm{~cm}$ & 81.2 & 81.5 & -0.3 & 1.8 & 1.9 & -0.1 & 3.567 & 3.527 & 1.1 \\
\hline $126 \mathrm{MeV}-3 \mathrm{~cm}$ & 81.2 & 81.5 & -0.3 & 1.8 & 1.9 & -0.1 & 3.808 & 3.773 & 0.9 \\
\hline
\end{tabular}

${ }^{\mathrm{a}} 14 \mathrm{~cm}$ square cerrobend aperture; $1,2,3 \mathrm{~cm}$ diameter circular brass collimators.

${ }^{\mathrm{b}}$ Percentage difference of peak-to-plateau ratio of SCDD with respect to that of IC. 


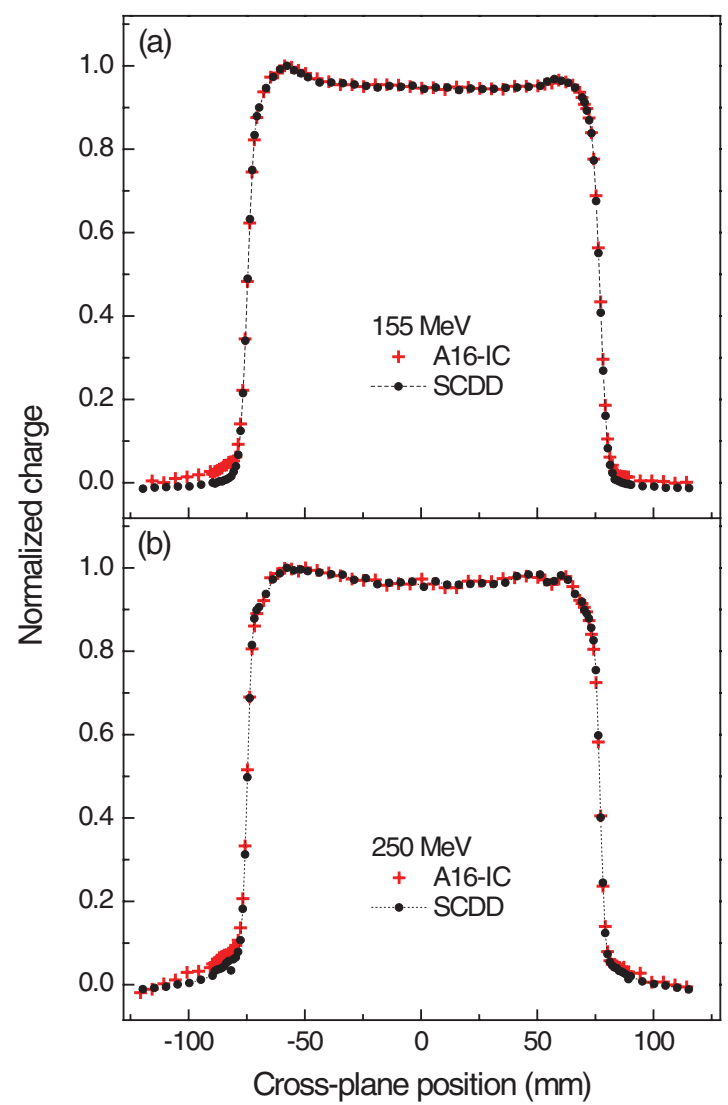

FIG. 9. Cross-plane profiles normalized at central axis dose measured by SCDD and A16-IC at a depth of $5 \mathrm{~cm}$ from the beam entrance window in the 155 and $250 \mathrm{MeV}$ proton beams with QA aperture.

by the diamond detector coincides with, or slightly overestimates, the Bragg peaks measured by ICs. Such a behavior is in complete contrast to what was reported in literature for thick natural diamond detectors which significantly underestimate the Bragg peaks purportedly attributed to a significant

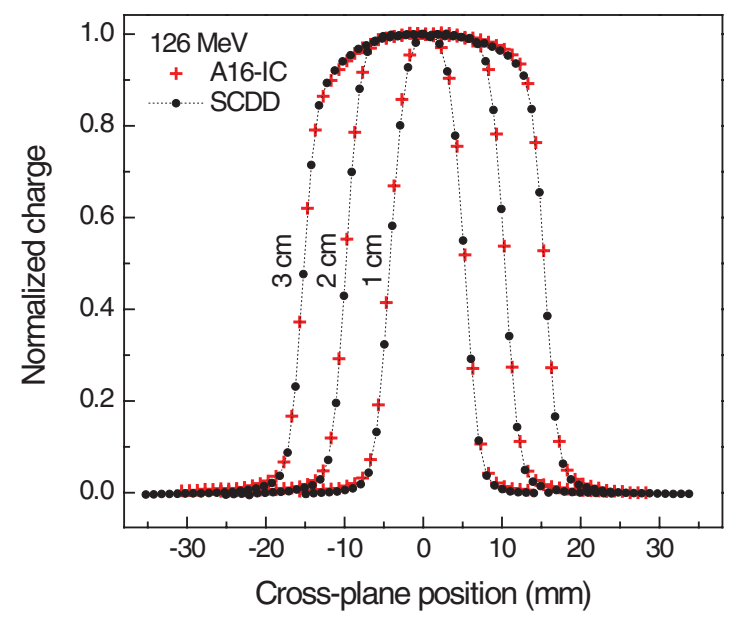

FIG. 10. Cross-plane profiles normalized at central axis dose measured by SCDD and A16-IC at a depth of $5 \mathrm{~cm}$ from the entrance window in the $126 \mathrm{MeV}$ proton beam with $1,2,3 \mathrm{~cm}$ in diameter circular brass collimators.
TABLE III. $80 \%-20 \%$ penumbra values from cross-plane profiles at $5 \mathrm{~cm}$ depth shown in Figs. 8 and 9.

\begin{tabular}{lccc}
\hline \hline & \multicolumn{2}{c}{$80 \%-20 \%$} & \\
penumbra (mm) & \\
$\begin{array}{c}\text { Proton energy - } \\
\text { size of aperture }\end{array}$ & SCDD & A16-IC & $\begin{array}{c}\text { difference } \\
\text { SCDD-A16-IC }\end{array}$ \\
\cline { 2 - 3 } $155 \mathrm{MeV}-14 \mathrm{~cm}$ & 4.9 & 5.1 & -0.2 \\
$250 \mathrm{MeV}-14 \mathrm{~cm}$ & 3.9 & 4.2 & -0.3 \\
$126 \mathrm{MeV}-1 \mathrm{~cm}$ & 2.7 & 2.7 & 0.0 \\
$126 \mathrm{MeV}-2 \mathrm{~cm}$ & 2.5 & 2.6 & -0.1 \\
$126 \mathrm{MeV}-3 \mathrm{~cm}$ & 2.8 & 2.9 & -0.1 \\
\hline \hline
\end{tabular}

a $14 \mathrm{~cm}$ square cerrobend aperture; $1,2,3 \mathrm{~cm}$ diameter circular brass collimators.

LET dependence and finite detector thickness. ${ }^{21,28,29}$ Such a dependence is not observed in the investigated dosimeter possibly due to the extremely reduced thickness of the active diamond layer $(1 \mu \mathrm{m})$. This is believed to minimize space charge formation and carrier recombination effects in the device, typically observed in high ionization density conditions.

\section{C. Beam profiles}

Figure 9 shows the normalized cross-plane profiles measured by SCDD and A16 microchamber for 155 and $250 \mathrm{MeV}$ proton beams (QA aperture). The profiles for the $126 \mathrm{MeV}$ proton beams with the 3 circular brass collimators are shown in Fig. 10. A good agreement between SCDD and A16-IC can be observed for all profiles. In Table III are listed the $80 \%-$ $20 \%$ penumbras from beam profiles by SCDD and A16-IC. For each profile, the tabulated penumbra values are the average of the left and right penumbras. The differences between SCDD and A16-IC measured penumbras are also shown in Table III. For the investigated energies/beam apertures, the SCDD gave smaller penumbra values than A16-IC, which means that SCDD has a slightly better spatial resolution than ionization chamber. This is consistent with geometric differences between the two detectors.

\section{CONCLUSIONS}

In the present work, a synthetic single crystal diamond detector fabricated at Roma "Tor Vergata" University laboratories has been tested and proven as a possible dosimeter for relative dosimetric measurements in high energy clinical proton beams.

The diamond dosimeter showed a stable and reproducible response. During the preirradiation procedure, a maximum deviation below $0.5 \%$ was found in the diamond detector signal from the average reading. A good linear behaviour was observed as a function of delivered dose over a dose range from 50 to $500 \mathrm{cGy}$, with a linearity index $\mathrm{R}^{2}$ equal to 1 and a precision of $10^{-6}$. The detector response was practically dose rate independent, with deviations of about $\pm 0.5 \%$ in the 85-300 cGy/min dose rates range. The detector showed very little stem effect, within $0.5 \%$. But the detector used in this study is a prototype of the commercially available 
synthetic diamond detector which is marketed and sold by PTW-Freiburg. The commercially available dosimeter differs from the prototype with regards to the housing and the encapsulation materials. The detector signal dependence on azimuthal angle is within $0.6 \%$. Fractional depth dose curves were in good agreement with those measured using reference dosimeters indicating a diamond response independent of the proton energy and LET. Lateral beam profiles measured by the diamond detector are in agreement with those obtained by the A16 microionization chamber. In particular, slightly sharper penumbras are obtained by the diamond dosimeter, indicating a good spatial resolution.

The observed dosimetric properties of the synthetic single crystal diamond detector indicate its suitability for accurate relative dosimetric measurements in large as well as in small field high- energy clinical proton beams.

\section{ACKNOWLEDGMENTS}

The authors wish to thank "Fondazione Roma" for financial support. This work was also jointly supported by EMRP within the Euramet project. The authors would also wish to thank Peter Koss and the accelerator operator crew.

a) Author to whom correspondence should be addressed. Electronic mail: bpatyal@llu.edu

${ }^{1}$ R. R. Wilson, "Radiological use of fast protons," Radiology 47, 487-491 (1946).

${ }^{2}$ J. M. Slater, J. O. Archambeau, D. W. Miller, M. I. Notarus, W. Preston, and J. D. Slater, "The proton treatment center at Loma Linda University Medical Center: Rationale for and description of the development," Int. J. Radiat. Oncol., Biol., Phys. 22, 383-389 (1992).

${ }^{3}$ B. Patyal, "Dosimetry aspects of proton therapy," Technol. Cancer Res. Treat. 6, 17-23 (2007).

${ }^{4}$ S. Braccini, "Progress in hadrontherapy," Nucl. Phys. B (Proc. Suppl.) 172, 8-12 (2007).

${ }^{5}$ IAEA (International Atomic Energy Agency), "Absorbed dose determination in external beam radiotherapy: An international code of practice for dosimetry based on standards of absorbed dose to water," Technical Report Series No. 398 (IAEA, Vienna, 2000).

${ }^{6}$ C. P. Karger, O. Jäkel, H. Palmans, and T. Kanai, "Dosimetry for ion beam radiotherapy,” Phys. Med. Biol. 55, R193-R234 (2010).

${ }^{7}$ ICRU (International Commission on Radiation Units and Measurements), "Prescribing, recording, and reporting proton-beam therapy," ICRU Report No. 78 (International Commission on Radiation Units and Measurements, Bethesda, MD, 2008).

${ }^{8}$ H. Palmans, F. Verhaegen, J. M. Denis, and S. Vynckier, "Dosimetry using plane parallel ionization chambers in a $75 \mathrm{MeV}$ clinical proton beam," Phys. Med. Biol. 47, 2895-2905 (2002).

${ }^{9}$ A. N. Schreuder, D. T. L. Jones, and A. Kiefer, "A small ionization chamber for dose distribution measurements in a clinical proton beam," in Advances in Hadrontherapy, edited by U. Amaldi, B. Larsson, and Y. Lemoigne (Elsevier, Amsterdam, 1977), pp. 284-289.

${ }^{10}$ M. Pacilio, C. De Angelis, S. Onori, L. Azario, A. Fidanzio, R. Miceli, A. Piermattei, and A. Kacperek, "Characteristics of silicon and diamond detectors in a $60 \mathrm{MeV}$ proton beam," Phys. Med. Biol. 47, N107-N112 (2002).

${ }^{11}$ V. S. Khrunov, S. S. Martynov, S. M. Vatnitsky, I. A. Ermakov, A. M. Chervjakov, D. L. Karlin, V. I. Fominych, and Yu. V. Tarbeyev,
"Diamond detectors in relative dosimetry of photon, electron and proton radiation Fields," Radiat. Prot. Dosim. 33, 155-157 (1990).

${ }^{12}$ S. Vatnitsky, D. Miller, J. Siebers, and M. Moyers, "Application of solid state detectors for dosimetry of therapeutic proton beams," Med. Phys. 22, 469-473 (1995)

${ }^{13}$ H. R. Lee, M. Pankuch, J. C. Chu, and J. J. Spokas, "Evaluation and characterization of parallel plate microchamber's functionalities in small beam dosimetry," Med. Phys. 29, 2489-2496 (2002).

${ }^{14}$ P. Francescon, S. Cora, and C. Cavedon, "Total scatter factors of small beams: A multi-detector and Monte Carlo study," Med. Phys. 35, 504-513 (2008).

${ }^{15}$ S. M. Vatnitsky, R. W. M. Schulte, R. Galindo, H. J. Meinass, and D. W. Miller, "Radiochromic film dosimetry for verification of dose distributions delivered with proton-beam radiosurgery," Phys. Med. Biol. 42, 1887-1898 (1997)

${ }^{16}$ D. Kirby, S. Green, H. Palmans, R. Hugtenburg, C. Wojnecki, D. Parker, "LET dependence of GafChromic films and an ion chamber in low-energy proton dosimetry," Phys. Med. Biol. 55, 417-433 (2010).

${ }^{17} \mathrm{O}$. A. Zeidan et al., "Dosimetric evaluation of a novel polymer gel dosimeter for proton therapy," Med. Phys. 37(5), 2145-2152 (2010).

${ }^{18}$ S. Safai, S. Lin, and E. Pedroni, "Development of an inorganic scintillating mixture for proton beam verification dosimetry," Phys. Med. Biol. 49, 4637-4655 (2004)

${ }^{19}$ ICRU (International Commission on Radiation Units and Measurements), "Clinical proton dosimetry: Part I. Beam production, beam delivery and measurement of absorbed dose," ICRU Report No. 59 (International Commission on Radiation Units and Measurements, Bethesda, MD, 1998).

${ }^{20}$ A. Fidanzio, L. Azario, C. De Angelis, M. Pacilio, S. Onori, A. Kacperek, and A. Piermattei, "A correction method for diamond detector signal dependence with proton energy," Med. Phys. 29, 669-675 (2002).

${ }^{21}$ S. Onori, C. De Angelis, P. Fattibene, M. Pacillo, E. Petetti, L. Azario, R. Miceli, A. Piermattei, L. Barone Tonghi, G. Cuttone, and S. Lo Nigro, "Dosimetric characterization of silicon and diamond detectors in lowenergy proton beams," Phys. Med. Biol. 45, 3045-3058 (2000).

${ }^{22}$ I. Ciancaglioni, Marco Marinelli, E. Milani, G. Prestopino, C. Verona, G. Verona-Rinati, R. Consorti, A. Petrucci, and F. De Notaristefani, "Dosimetric characterization of a synthetic single crystal diamond detector in clinical radiation therapy small photon beams," Med. Phys. 39, 4493-4502 (2012).

${ }^{23}$ C. Di Venanzio, Marco Marinelli, E. Milani, G. Prestopino, C. Verona, G. Verona-Rinati, M. D. Falco, P. Bagalà, R. Santoni, and M. Pimpinella, "Characterization of a synthetic single crystal diamond schottky diode for radiotherapy electron beam dosimetry," Med. Phys. 40, 021712 (9pp.) (2013).

${ }^{24}$ M. F. Moyers, "Proton therapy," in The Modern Technology of Radiation Oncology: A Compendium for Medical Physicists and Radiation Oncologists, edited by J. Van Dyk (Medical Physics, Madison, Wisconsin, 1999), pp. 823-869.

${ }^{25}$ S. Almaviva, M. Marinelli, E. Milani, G. Prestopino, A. Tucciarone, C. Verona, G. Verona-Rinati, M. Angelone, M. Pillon, I. Dolbnya, K. Sawhney, and N. Tartoni, "Chemical vapor deposition diamond based multilayered radiation detector: Physical analysis of detection properties," J. Appl. Phys. 107(1), 014511-014517 (2010).

${ }^{26}$ K. P. Gall et al. "State of the Art? New proton medical facilities for the Massachusetts General Hospital and the University of California Davis Medical Center," Nucl. Instrum. Methods Phys. Res. B 79, 881-884 (1993).

${ }^{27}$ B. Gottschalk, On the characterization of spread out Bragg peaks and the definition of "Depth" and "Modulation" http://huhepl.harvard.edu/ $\sim$ gottschalk.

${ }^{28}$ G. A. P. Cirrone, G. Cuttone, L. Raffaele, M. G. Sabini, C. De Angelis, S. Onori, M. Pacilio, M. Bucciolini, M. Bruzzi, and S. Sciortino, "Natural and CVD type diamond detectors as dosimeters in hadrontherapy applications," Nucl. Phys. B (Proc. Suppl.) 125, 179-183 (2003).

${ }^{29}$ H. Bichsel, "Calculated Bragg curves for ionization chambers of different shapes,” Med. Phys. 22, 1721-1726 (1995). 